\title{
CHALLENGES IN THE APPLICATION OF GEOMETRIC CONSTRAINT MODELS
}

5

\author{
Craig R. McClain ${ }^{1 *}$, Ethan P. White ${ }^{2,3}$, and Allen H. Hurlbert ${ }^{4}$
}

10

15

20

$$
\begin{gathered}
{ }^{1} \text { Monterey Bay Aquatic Research Institute, } \\
7700 \text { Sandholdt Road, Moss Landing, CA 95039, USA } \\
\text { Email: cmclain@mbari.org } \\
{ }^{*} \text { Corresponding author }
\end{gathered}
$$

${ }^{2}$ Department of Ecology and Evolutionary Biology, University of Arizona, Tucson, AZ 85721, USA

${ }^{3}$ Department of Biology, Utah State University, Logan, UT, 84322, USA

${ }^{4}$ National Center for Ecological Analysis and Synthesis 735 State Street, Suite 300, Santa Barbara, CA 93101, USA

25 Keywords: mid-domain effect, geometric constraint model, null model, debate, species’ range, species richness, diversity 


\begin{abstract}
Discerning the processes influencing geographic patterns of species richness remains one of the central goals of modern ecology. Traditional approaches to exploring these patterns have

30 focused on environmental and ecological correlates of observed species richness. Recently, some have suggested these approaches suffered from the lack of an appropriate null model that accounts for species' ranges being constrained to occur within a bounded domain. Proponents of these null geometric constraint models (GCMs), and the mid-domain effect these models produce, argue their utility in identifying meaningful gradients in species richness. This idea has

35 generated substantial debate. Here we discuss what we believe are the three major challenges in the application of GCMs. First, we argue there are actually two equally valid null models for the random placement of species ranges within a domain, one of which actually predicts a uniform distribution of species richness. Second, we highlight the numerous decisions that must be made to implement a GCM that lead to marked differences in the predictions of the null model.

40 Finally, we discuss challenges in evaluating the importance of GCMs once they have been implemented.
\end{abstract}

\title{
INTRODUCTION
}

In short, the recent emphasis on the need to evaluate perceived patterns in community structure against null hypotheses is - as seen with hindsight overdue. But legitimate enthusiasm for sound methodology must go hand in hand with the realization that null hypotheses in ecology, as elsewhere, depend on null models, and that all models make assumptions. If these assumptions are not appropriate, or create systematic biases, no amount of mathematical and statistical precision will produce biologically valid answers. - Harvey, Colwell, Silvertown, and May (1983) 
The application of null models has a controversial history in ecology (Gotelli \& Graves, 1996). One of the most recent of these debates centers on the mid-domain effect and geometric

55 constraint models (GCMs, e.g. Cowell et al., 2005; Hawkins et al., 2005; Zapata et al., 2005). These null models generate patterns of species richness in the absence of geographical gradients in ecological processes that affect richness, taking into account the naturally restricted area in which species can occur. In general GCMs produce a central peak in species richness when species ranges are randomly placed within a bounded domain, and it has been argued that these

60 stochastic models can in part explain such classic ecological patterns as the latitudinal gradient in species richness (Colwell \& Hurtt, 1994; Willig \& Lyons, 1998; Colwell \& Lees, 2000).

In general, null models are designed to determine if the observed pattern differs from expectations in the absence of some important process (Harvey et al., 1983; Gotelli \& Graves, 1996). In the case of GCMs, this is actually a large group of processes - all non-random

65 processes predicted to generate gradients in species richness (Colwell \& Hurtt, 1994; Colwell \& Lees, 2000; Colwell et al., 2004). Having decided which processes to exclude, the challenge is to design a procedure that will produce the pattern anticipated in the absence of those processes. However, a single best choice of procedure is not always obvious (Gotelli, 2000) making inferences from this type of approach more difficult. Here we suggest that at the present time

70 GCMs suffer from three challenges in this regard: 1) uncertainty regarding the basic mode of random range placement and the consequent null pattern of richness that would be expected (i.e., a mid-domain peak or uniform richness) in the absence of the identified processes; 2) uncertainty about how to generate the null pattern, even when the basic mode of range placement is agreed upon; and 3) uncertainty regarding how to best evaluate the importance of GCMs in explaining 75 empirical richness patterns. 


\section{HOW SHOULD RANGES BE RANDOMLY PLACED IN THE ABSENCE OF ECOLOGICAL PROCESSES?}

The null hypothesis tested in any analysis of biogeographical data... is not that empirical patterns do not differ from random ones, but that they do not differ from patterns generated by a particular model of the world. Colwell and Winkler (1984)

Colwell and Hurtt (1994) originally proposed two general classes of null models for patterns of species richness generated by randomly distributed species ranges. Both of these null models involve placing a geographic range randomly onto a domain (envision throwing disks of varying size onto a mat on the floor). In the first model (their model 1), if a range overlaps a domain boundary the portion of that range that lies outside the boundary is cut off. As such, the

90 center of a terrestrial species' range could occur in the middle of an ocean, with only a small portion of the randomly placed range actually occurring on land. Colwell and Hurtt (1994) considered this model to represent a "baseline against which to evaluate the effect of introducing additional assumptions in the models that follow (p. 572).” In the second type of model (their models 2 and 3, hereafter geometric constraint models, or GCMs), if a range falls on a boundary

95 it is picked up and thrown again until it falls entirely within the range (see Colwell \& Hurtt, 1994 and Sandel \& McKone, 2006 for more detailed descriptions of these models). These two types of null models are both logically valid null models for the random distribution of ranges in a bounded domain. However, they make very different assumptions about how the "random" placement of ranges operates - model 1 suggests that there are no boundary constraint effects on species richness, whereas GCMs assume substantial boundary effects. As a result these different types of null model also predict very different diversity patterns - model 1 predicts that species 
richness will be uniformly distributed across the landscape, while GCMs predict a peak in richness at the center of the domain (Sandel \& McKone, 2006).

The existence of two logically valid null models for random range placement poses a 105 problem for unambiguously testing the null expectation. Are there reasons to choose one model over another? Colwell and Hurtt (1994) rejected the first model based on deviations of observed data from secondary predictions of the model. Specifically, Colwell and Hurtt (1994) noted, "If a hard-boundary version of [model 1] were realistic, we would expect a drop in mean latitudinal range and a stacking up of range limits at the northern extremes of these distributions... Not only

110 is there no such pattern, but mean latitudinal range actually peaks at or near the northern limit for most groups, as Stevens (1989) points out, and range limits do not tend to stack up (G. C. Stevens personal communication)”.

However, one cannot characterize a null model as being inappropriate because it does not reproduce empirically observed patterns. The purpose of a null model is to represent the

115 observed pattern in the absence of some chosen process, and differences between the null model predictions and the data suggest that the relevant process is in fact operating. Thus, the appropriateness of a null model should be judged on its assumptions rather than on comparisons between the model output and the data. Were we to only identify as valid, null models that matched the data in all aspects, then by definition reality would always confirm the null expectation. Sandel and McKone’s (2006) recent argument that these secondary patterns can be used to "help in assessing the usefulness of the various models" suffers from the same logical fallacy. In this case, Colwell and Hurtt (1994) have argued that the fact that: 1) range edges do not "stack up" in northern latitudes, and 2) average range area increases in northern latitudes, is evidence against the placement of ranges in accordance with model 1. However, it is clearly 
125 possible to generate these same empirical patterns by placing ranges according to model 1 while simultaneously imposing a gradient in species richness and/or average range size.

The confusion regarding the rejection of model 1 based on these secondary characteristics likely resulted because the predictions of both types of null models are contingent upon two assumptions: 1) the type of range placement, and 2) the absence of meaningful ecological

130 gradients that affect richness. Therefore, deviations from the predictions of the model can occur either because the assumptions about the type of random range placement are wrong (as Colwell and Hurtt, 1994 assert) or because of the presence of a meaningful gradient in species richness. Since a gradient in species richness is typically what is being investigated in these types of studies, deviations from a null model that predicts an absence of gradients (or associated 135 secondary characteristics) offer no evidence against using model 1 as a reasonable null. Moreover, model 1 is not unique in failing to reproduce all aspects of patterns. GCMs also suffer from secondary inconsistencies with empirically observed data. These include a lack of symmetric unimodality in most observed data (Willig \& Lyons, 1998; Jetz \& Rahbek, 2001; Laurie \& Silander, 2002; McClain \& Etter, 2005), a mismatch to observed patterns of spatial 140 turnover (Koleff \& Gaston, 2001), and predicted zero values for species richness at the edges of domains (Laurie \& Silander, 2002). As such, neither null model can be characterized as superior with respect to reproducing all secondary patterns.

In addition to the arguments regarding secondary characteristics, Colwell and Hurtt (1994) also suggest that GCMs may be more appropriate than model 1 based on "theories of 145 extinction and minimum viable population size.” Ranges cannot become arbitrarily small at domain boundaries because (ceteris paribus) there will be some minimum range size below which a population is unlikely to persist as a result of demographic stochasticity. While this may 
represent a reasonable argument against the strict implementation of model 1 , it makes no distinction between GCMs and a version of model 1 that includes a minimum range size. This would result in a hybrid model, with a uniform distribution of richness across most of the domain and a decrease in richness only near the edges. When the minimum viable range size is small compared to the domain, as would be expected for most continental domains, such a model would be mostly indistinguishable from model 1. In addition, Sandel and McKone (2006) have recently argued that model 1 (their ‘truncation' model) is the more appropriate null based on the

155 idea that species tend to occur under particular sets of environmental conditions. If the domain were somehow expanded it is possible that species at the domain's edge would encounter additional suitable habitat and have larger realized ranges. In other words, the observed range of a species that abuts the domain edge may only represent a small portion of it's 'potential range' (Sandel \& McKone, 2006).

Therefore at least two (more if one considers potential hybrid models) equally viable classes of null model exist for patterns of species richness in the absence of environmental gradients. To implement the first of Colwell and Hurt's (1994) null models no additional decisions need to be made. The expectation in the absence of geographic gradients is simply a uniform distribution of species richness, as has been assumed implicitly by studies that do not

165 address the 'mid-domain effect'. However, to implement GCMs, a variety of decisions must be made, and as we summarize in the subsequent sections, these choices will affect the null expectation.

\section{IF RANGE PLACEMENT IS BOUNDED, HOW SHOULD IT BE EXECUTED?}


problem such as population growth in a resource-limited environment can be addressed with a variety of analytical tools, none of which provides a single "right" answer to the question... Similarly, many different null models can be constructed to generate community patterns in the absence of interspecific competition... The diversity of null models has not always been appreciated. - Gotelli and Graves (1996)

If a GCM is to be used as the null model for the random distribution of species’ ranges, then a number of decisions must be made during model implementation (Colwell et al. 2004). Each decision actually constitutes choosing a slightly different null model (Gotelli, 2000), and understanding which null model is appropriate to answer a given question is important. Unfortunately, in many cases there is no obviously correct choice regarding how the model

185 should be implemented due to our limited understanding of the biology of geographic range formation, but these decisions must be made nonetheless. The aim of this section is not to provide a comprehensive review, nor in most cases to provide advice about which choices to make, but simply to illustrate the types of decisions that need to be made, the current debate regarding the most appropriate approaches to dealing with them, and the potential effects of the different decisions on predicted richness patterns.

\section{Dimensionality}

For historical reasons of perception, data availability, and the feasibility of analysis, the complex two-dimensional pattern of species richness on the globe has often been characterized as sets of one-dimensional gradients (Hawkins \& Diniz-Filho, 2004). Mid-domain models were

195 initially developed in this context as null models for one-dimensional latitudinal, elevational, and bathymetric gradients (Colwell \& Hurtt, 1994; Pineda \& Caswell, 1998), and consequently the great majority (>90\%) of studies examining geometric constraints have done so in only a single dimension (Colwell et al., 2004). Despite the role of latitude in inspiring GCMs, a mid-domain 
effect is expected for any arbitrary transect across a domain. In the absence of environmental

200 gradients, a peak in richness should occur not just at the latitudinal midpoint of a domain, for example, but at the longitudinal midpoint, the NW-SE midpoint, et cetera. While this logic seems straightforward there is still debate in the literature over whether 1-D, 2-D, or a combination of the two is most appropriate (Bokma et al., 2001; Diniz-Filho et al., 2002; Hawkins \& Diniz, 2002; Zapata et al., 2003; Colwell et al., 2004). In addition there are gradients that may be more naturally characterized in a single dimension such as depth, elevation, rivercourses, etc., and there may be reason to include three dimensional versions of continental GCMs that incorporate variability in elevation (Colwell et al., 2004).

\section{Range Placement Method}

One of the first decisions to be made is how to place ranges within the bounded domain

210 (Colwell \& Hurtt, 1994; Sandel \& McKone, 2006). Even in the simple 1-D case a number of methods to randomly place ranges within the domain exist (Connolly 2005) and expanding into two dimensions complicates the matter further. The primary methods for randomly placing ranges in either 1-D or 2-D cases are: 1) range shuffling - ranges are randomly placed such that they lie entirely within the domain (the pencil box analogy of Colwell et al., 2004); 2) spreading

215 dye - a random starting point is chosen within the domain and the range spreads until it has reached the appropriate size by randomly filling unoccupied cells adjacent to the range edge while remaining within the domain (Jetz \& Rahbek, 2001); and 3) Poisson placement - where range expansion from a starting point is governed by a Poisson probability of halting expansion (Connolly, 2005). While all of these approaches are reasonable and intuitive they can produce

220 noticeably different null expectations based on details of the underlying implementation (Connolly, 2005; Sandel \& McKone, 2006). 


\section{Choosing a Range Size Frequency Distribution}

The choice of an appropriate range size frequency distribution (RSFD) greatly affects the expected richness pattern of any geometric constraint model and therefore the goodness of fit to

225 empirical richness data (Colwell \& Hurtt, 1994; Laurie \& Silander, 2002; Colwell et al., 2004). There are typically two choices: 1) range sizes and midpoints may be drawn from a uniform distribution of all possible combinations (i.e. all combinations occurring entirely within the domain); or 2) the RSFD may be constrained to match that of the observed data. The decision regarding which RSFD to use is a decision about how much the null model should be constrained

230 by empirical data. Colwell and colleagues (2004; 2005) have recently argued strongly for using the observed RSFD, contending that the most appropriate null model should incorporate all aspects of biology (including the observed RSFD) while excluding only the factor of interest (i.e., non-random range placement). Others have argued that biological factors that may shape the observed RSFD may be “smuggled in” to the null model, leading to an overestimation of the

235 importance of geometric constraints alone (Zapata et al., 2003; Connolly, 2005; Hawkins et al., 2005). This debate over which RSFD is most appropriate for geometric constraints models is analogous to the debate over non-random community assembly over the past several decades. While some authors argued that the appropriate null involves randomly filling the species-bylocation matrix with the appropriate number of occupancies, others suggested that either row

240 sums or column sums or both must be maintained in the randomization (Colwell et al., 2005). These different choices amount to the inclusion of different assumptions about processes occurring in the system (Harvey et al., 1983) and can have marked impacts on the outcome and interpretation of the study (e.g., Gotelli, 2000).

\section{Determining the Domain Limits}


In order to conduct a GCM analysis it is necessary to define the relevant boundaries for the system being studied and the question being addressed. Colwell and Lees (2000) proposed four types of domain limits: 1) continental edges; 2) ecological regions of sub-continental scale (e.g., biomes); 3) distributional limits of the focal taxon; and 4) arbitrary boundaries (e.g., $20^{\circ} \mathrm{N}$ to $20^{\circ} \mathrm{S}$ latitude). Arguments both for (Colwell \& Lees, 2000; Colwell et al., 2004) and against

250 (Koleff \& Gaston, 2001; Zapata et al., 2005; Kerr et al., 2006) the different types of boundaries are present in the literature. However, generally speaking, the choice of domain boundaries is often not clear or intuitive, and this uncertainty is problematic given the dependency of the predicted richness peak on the exact boundary locations (Vetass \& Grytnes, 2002; McClain \& Etter, 2005). This phenomenon is well illustrated by Willig and Lyons (1998) who examine the

255 match between empirical patterns of richness in New World marsupials and GCM predictions using three different latitudinal domains: the full latitudinal extent of the New World (disagreement in peak location, poor fit), the latitudinal extent of the marsupial fauna (agreement in peak location, poor fit), and the smallest latitudinal extent to include 95\% of the marsupial species (agreement in peak location, good fit). Depending on the choice of boundaries very

260 different conclusions may be reached about the importance of geometric contrains.

\section{Including Non-endemic Species}

The logic of GCMs is contingent upon the examination of species endemic to the domain (Colwell \& Lees, 2000; Jetz \& Rahbek, 2001; Whittaker et al., 2001; Hawkins \& Diniz-Filho, 2002; Colwell et al., 2004; Pimm \& Brown, 2004). However, the fact that non-endemic species

265 exist and therefore must be dealt with in analyses creates confusion and ambiguity for choosing the appropriate null model. As the percentage of non-endemic species in the biota increases (e.g., biomes will have proportionally more non-endemics than continents), this issue becomes 
increasingly important. Three approaches have been proposed for dealing with non-endemics: 1) exclude them entirely from all analyses (Colwell \& Lees, 2000; Jetz \& Rahbek, 2001); 2)

270 truncate the range at the domain edge and treat the portion occurring within the domain as an endemic (Diniz-Filho et al., 2002; McCain, 2003); and 3) truncate the range at the domain edge, and force these non-endemic range fragments to remain attached to a domain edge during randomization (Colwell et al., 2004). Colwell et al. (2004) have argued that this decision does not strongly affect GCM fits to the data (based solely on correlation coefficients), but it can

275 certainly affect the general shape and magnitude of the predicted relationship (e.g., the third approach will clearly produce flatter richness peaks than the second approach), especially in cases where the number of non-endemics is large.

\section{Range porosity}

One assumption of classic GCMs is that species are found at all localities within their

280 geographic range. However, most species’ ranges are patchily occupied (Rapoport, 1982;

Gaston, 2003; Hurlbert \& White, 2005), and this patchiness has potential consequences for the implementation of GCMs (Hawkins et al. 2005). Pineda and Caswell (1998) deal with this by incorporating the actual patchiness of species distributions in the null predictions by randomizing the species by location matrix for point samples. However, this assumes a complete lack of

285 meaningful range coherence, which is clearly not the case in natural systems (Rahbek and Graves, 2000; Colwell et al., 2004). Alternatively, McClain and Etter (2005) varied the percentage of species randomly removed from point samples after ranges had been shuffled along a bathymetric gradient. While only at severe "porosity” was the mid-domain peak eliminated (see also Zapata et al., 2003; Colwell et al., 2004), the magnitude and curvature of the 290 predicted richness curve varied systematically with mean range porosity (McClain \& Etter, 
2005). Colwell et al. (2004) argue that range porosity is "rarely a source of significant bias for most taxa” because ranges are generally not particularly porous and range contiguity would have to be near zero to completely remove the mid-domain richness peak. However, recent work on over 300 bird species has shown that on average species only occur on 50-60\% of the surveys

295 within their range (Hurlbert \& White, 2005). While this level of range porosity does not eliminate the mid-domain peak, it does have substantial effects on GCM model predictions (McClain \& Etter, 2005), and should thus be explicitly modeled when possible (e.g. Dunn et al., 2006). In addition, the fact that survey data reflect patterns at a relatively fine resolution with very little spatial autocorrelation remaining after accounting for environmental factors (Hurlbert \& White, 2005) suggests that the rationale for applying GCMs to survey data is generally weaker.

In general, this section demonstrates that there are a large number of decisions to be made when implementing a GCM. In some cases there are strong, but contradictory opinions about the most appropriate course of action. In other cases, there is simply a recognition that a decision

305 must be made, but little suggestion for which approach, if any, is superior. Given the fairly substantial affects that some of these decisions have on the predictions of the null model (e.g., Willig \& Lyons, 1998; Grytnes \& Vetaas, 2002; Bellwood et al., 2005; Connolly, 2005; McClain \& Etter, 2005) this represents a significant challenge for the implementation and interpretation of GCMs. In other words, if it is deemed necessary to include a GCM in an analysis of species 310 richness patterns, which GCM is it that should actually be used?

\section{EVALUATING THE IMPORTANCE OF GEOMETRIC CONSTRAINTS}

Part of the confusion over how best to evaluate GCMs stems from the historical context in which null models were developed. Community null models of the 1970s and 1980s were used 
to ask some version of the question, "Do ecologically or taxonomically similar species co-occur

315 less frequently than expected by chance?” Such null models relied on the principle of falsification (Gotelli \& Graves, 1996), categorizing empirical patterns as being either distinguishable or indistinguishable from random based on some metric of co-occurrence. The focus of GCMs, on the other hand, is on the spatial pattern of species richness, an inherently complex pattern which cannot be reduced to a single metric. As such, authors have evaluated

320 GCMs in a number of different ways including: 1) overall model fit based on correlation (e.g., Willig \& Lyons, 1998); 2) testing a plot of predicted versus observed richness values for a slope of 1 and intercept of 0 (e.g., Jetz \& Rahbek, 2001; Romdal et al., 2005); 3) comparison of empirical richness values to 95\% confidence intervals around GCM predictions (e.g., McCain, 2003); 4) comparison of curve parameters such as curvature, height, and peak location (e.g.,

325 McClain \& Etter, 2005); 5) evaluating the degree to which the null model accounts for secondary predictions such as species turnover and the positions of geographic ranges (e.g., Koleff \& Gaston, 2001); and 6) testing GCM predictions when potential environmental determinants of richness are controlled for (Jetz \& Rahbek, 2002).

Some of these methods of evaluation are hypothesis tests in the tradition of earlier null 330 models that ask whether the entire pattern (method 2) or some aspect of it (method 4) differs from the null expectation. Despite this tradition of falsification in community null models, Colwell et al. (2004) argue against using such an "all-or-nothing” approach. This is understandable given that the complexity of the pattern being predicted allows for the 'partial matching' of null model predictions. Along these lines Colwell and Lees (2000, p. 75) suggest 335 that "Departure from the expected richness peak, under an appropriate null model, but not the peak itself, requires biological or historical explanation...” Connolly et al. (2003) discuss and 
interpret patterns of fish and coral diversity in just this way, focusing on deviations from the null predictions. However, this approach assumes that GCM predictions have logical primacy (see Roughgarden, 1983 for a discussion of logical primacy and null models) over other candidate 340 predictor variables in explaining richness patterns. This can be problematic as can be seen by considering the case where richness increases linearly across a domain as a direct result of some gradient. Since GCM predictions are by definition hump-shaped, are we to seek out ecological variables that explain the J-shaped pattern of the residuals or the simpler linear relationship (Figure 1)? Furthermore, one could envision a scenario in which richness varies across the 345 domain in a manner consistent with the predictions of a GCM, and yet the ranges are distributed in a distinctly non-random, perfectly nested manner (Figure 2). While this nested distribution of ranges begs for a biological explanation (e.g., nested physiological tolerances, unimodal variation in resource availability with nested competitive abilities, etc.), a strong correlation between GCM predictions and the empirical pattern would suggest there was nothing further to 350 explain.

More recently, Colwell et al. (2004, p. E14) stated that "there is no justification for treating [mid-domain effect] predictions as primary just because they are generated by a null model.” Instead, they argue that GCM predictions should be treated identically to other ecological variables and included in a multivariate framework. Thus far only a few studies

355 examining GCMs have simultaneously considered other physical, biological, or historical correlates of richness (e.g., Jetz \& Rahbek, 2002; Bellwood et al., 2005; Mora \& Robertson, 2005; Watkins et al., 2006). This approach alleviates the types of problems illustrated in Figure 1 and thus is clearly preferable as pointed out by Colwell et al. (2004). However, the use of regression models evaluates the fit or partial fit of GCM predictions in a relative rather than 
360 absolute sense (Zapata et al., 2003; Colwell et al., 2004). Predicted richness and empirical richness can be highly correlated and yet substantially different in magnitude, and thus such analyses overestimate the importance of GCMs. Environmental correlates do not have this problem because coefficients for these variables are intentionally being fitted to the data in order to determine the model (i.e., environmental models have free parameters whereas GCMs by

365 definition do not). As a result of this difference GCM predictions need to be treated differently than environmental variables and a method for integrating these two distinct types of predictors has not yet been proposed.

One area of analysis of GCMs that has been almost entirely ignored is consideration of power. Power is the probability of detecting a non-random (or non-null) pattern when one is

370 actually present. Knowing the power of null models is important, because a null model with very low power will almost always suggest that non-random processes are not operating, even when they are operating quite strongly (e.g., Toft \& Shea, 1983; Losos et al., 1989; Kelt et al., 1995; Gotelli, 2000). In a multivariate framework, power determines how easily the effects of different predictor variables can be distinguished from one another. For example, one-dimensional

375 evaluations of richness patterns have inherently less power than two-dimensional analyses because of a simplification of pattern and concomitant loss of information. Collapsing variables and predictions onto a single dimension is likely to increase multicollinearity, reducing the ability to distinguish among variables. For example, were we to partition the variance in latitudinal species richness explained by net primary productivity and a one-dimensional GCM 380 we would likely find that much of this variance cannot be uniquely ascribed to either variable, greatly confounding the interpretation of these results. Detailed analyses of power are necessary to understand the strength with which the absence of process can be inferred from GCM results. 
Along these lines, it is worth pointing out that if one-dimensional simplifications are used for studying GCMs then the strength of the MDE should be approximately equal for all onedimensional axes that cross the center of the domain (ceteris paribus). Thus, support for a GCM could be based on the strength of the weakest one-dimensional relationship, or perhaps on some measure of the central tendency of the distribution of strengths across all possible axes (e.g. the average correlation coefficient). If a GCM explains $80 \%$ on only one axis but little on most of the others, then the logical inference is that there is only weak evidence for a MDE, and that environmental variables that correlate with the primary axis are candidate explanatory richness variables. This observation raises serious questions about support for GCMs drawn from 1-D analyses that have focused solely on a single axis for which a strong gradient is known to exist.

\section{CONCLUSIONS}

In this paper we have highlighted three major challenges in the current application of null models for species richness. The first, and perhaps most critical of these, is that the primary published justification for choosing GCMs over the simpler Type 1 null model is flawed. A null model cannot be rejected because it does not match empirical patterns. The null model must be chosen because it is appropriate for the question being asked, and deviations from that model

400 indicate that the excluded process (or processes) is in fact operating. Unfortunately, there is no way to know which of the two general types of null model presented by Colwell and Hurtt (1994), or potential hybrids, is in fact justified by the question being asked: "what patterns of species richness would arise under minimal biological assumptions, in a world without geographical gradients in adaptation, speciation rate, extinction rate, habitat diversity,

405 productivity, competition, predation, or dispersal”? (p. 570). As such, until we better understand 
the processes responsible for geographic range formation and how they would operate in the presence of boundaries to range expansion, the choice of null model is a matter of preference rather than objective reasoning.

The second challenge is the fact that even if GCMs are considered to be the most 410 appropriate general type of null model, the exact null expectation is contingent on a number of choices that must be made during model implementation. These choices can lead to substantially different null predictions for species richness patterns (Bellwood et al., 2005; Connolly, 2005; McClain \& Etter, 2005; Sandel \& McKone, 2006). Therefore there does not exist a single null prediction, but a suite of them, and there is rarely a concrete justification for choosing some 415 realizations over others (Colwell et al., 2004). One is left to make these choices arbitrarily, or one could be tempted to make choices that maximize the fit to the data. Neither of these approaches represents an objective test of a specific null hypothesis. At the minimum researchers should detail the specific decisions made in implementing a GCM.

Finally, even if one particular method of implementing GCMs could be viewed as 420 superior, it is not clear how best to evaluate these types of randomization based null models and their contributions to observed patterns. There is significant disagreement in the literature over whether GCMs: 1) should be treated like more traditional null hypotheses and either accepted or rejected (Pineda \& Caswell, 1998; Connolly et al., 2003), or whether their predictions should be included as predictive variables in multivariate analyses (Colwell et al., 2004; 2005); 2) should

425 be given logical primacy (Colwell \& Lees, 2000; Bokma et al., 2001) or treated as equivalent to other variables (Colwell et al., 2004; 2005); and 3) should be evaluated using secondary predictions (Koleff \& Gaston, 2001; Laurie \& Silander, 2002; Connolly et al., 2003) or only based on species richness patterns per se (Colwell et al., 2004). In addition, there are problems 
related to power and multicollinearity between null model predictions and relevant

430 environmental variables. At the moment, there is no straightforward way to deal with these kinds of issues.

In general, we have sought to highlight the fact that random range placement models can be carried out in a variety of different ways, depending upon assumptions regarding the basic nature of the null, the inclusion of non-endemics, the definition of the domain, the

435 dimensionality of the analysis, etc. Not all such models will predict a peak in richness at the center of the domain - some will predict uniform richness - and among those that do predict a peak there will be differences in the specific form of the predicted richness pattern. This suggests that the 'mid-domain effect' per se is neither as clean nor as universal a null hypothesis as has sometimes been suggested and indicates that substantial additional work is necessary before real 440 inference can be drawn from GCM analyses.

\section{ACKNOWLEDGEMENTS}

CRM was supported by NSF Biocomplexity grant \#DEB-0083422 and a Monterey Bay Aquatic Research Institute Postdoctoral Fellowship. EPW was supported by an NSF Graduate Research Fellowship and an NSF Postdoctoral Fellowship in Bioinformatics. AHH was supported by NSF 445 Biocomplexity grant \#DEB-0083422 and the National Center for Ecological Analysis and Synthesis. We thank Jeffrey Nekola, Bradford Hawkins, Mike Weiser and Walter Jetz for their insightful comments on the manuscript. Finally, the manuscript has benefited substantially from an in-depth review by Robert Colwell who, while he might not agree with all of our positions, has helped us to clarify our statements and to ensure that we represent both sides of the debate as 450 accurately as possible. 


\section{LITERATURE CITED}

Bellwood, D.R., Hughes, T.P., Connolly, S.R. \& Tanner, J. (2005) Environmental and geometric constraints on Indo-Pacific coral reef biodiversity. Ecology Letters, 8, 643-651.

Bokma, F., Bokma, J. \& Monkkonen, M. (2001) Random processes and geographic species richness patterns: why so few species in the north? Ecography, 24, 43-49.

Colwell R.K. (2006) RangeModel: A Monte Carlo simulation tool for assessing geometric constraints on species richness. Version 5. User's Guide and application published at: http://viceroy.eeb.uconn.edu/rangemodel.

Colwell R.K., \& Hurtt, G.C. (1994) Nonbiological gradients in species richness and a spurious Rapoport effect. American Naturalist, 144, 570-595.

Colwell, R.K. \& Lees, D.C. (2000) The mid-domain effect: geometric constraints on the geography of species richness. Trends in Ecology and Evolution, 15, 70-76.

Colwell, R.K., Rahbek, C. \& Gotelli, N.J. (2004) The mid-domain effect and species richness patterns: what have we learned so far? American Naturalist, 163, E1-E23

465 Colwell, R.K., Rahbek, C. \& Gotelli, N.J. (2005) The mid-domain effect: There's a baby in the bathwater. American Naturalist, 166, E149-E154.

Colwell, R.K. \& Winkler, D.W. (1984) A null model for null models in biogeography. In: Ecological communities: conceptual issues and the evidence (eds. Strong DR, Simberloff D, Abele LG \& Thistle AB), pp. 344-359. Princeton University Press, Princeton, NJ.

470 Connolly, S.R. (2005) Process-based models of species distributions and the mid-domain effect. American Naturalist, 166, 1-11.

Connolly, S.R., Bellwood, D.R. \& Hughes, T.P. (2003) Indo-Pacific biodiversity of coral reefs: Deviations from a mid-domain model. Ecology, 84, 2178-2190. 
Connolly, S.R., Hughes, T.P., Bellwood, D.R. \& Karlson, R.H. (2005) Community structure of 475 corals and reef fishes at multiple scales. Science, 309, 1363-1365.

Diniz-Filho, J.A., de Sant'Ana, C.E.R., de Souza, M.C. \& Rangel, T. (2002) Null models and spatial patterns of species richness in South American birds of prey. Ecology Letters, 5, 47-55.

Dunn, R.R., Colwell, R.K., \& Nilsson, C. (2006) The river domain: why are there more species 480 halfway up the river? Ecography, 29, 251-259.

Gaston, K.J. (2003) The Structure and Dynamics of Geographic Ranges. Oxford University Press, Oxford.

Gotelli, N.J. (2000) Null model analysis of species co-occurrence patterns. Ecology, 81, 26062621.

485 Gotelli, N.J. \& Graves, G.R. (1996) Null models in ecology. Smithsonian Institution Press, Washington DC.

Grytnes, J.A. \& Vetaas, O.R. (2002) Species richness and altitude: A comparison between null models and interpolated plant species richness along the Himalayan altitudinal gradient, Nepal. American Naturalist, 159, 294-304.

490 Harvey, P.H., Colwell, R.K., Silvertown, J.W. \& May, R.M. (1983) Null models in ecology. Annual Review of Ecology and Systematics, 14, 189-211.

Hawkins, B.A. \& Diniz-Filho, J.A.F. (2002) The mid-domain effect cannot explain the diversity gradient of Nearctic birds. Global Ecology and Biogeography, 11, 419-426.

Hawkins, B.A. \& Diniz-Filho, J.A.F. (2004) 'Latitude' and geographic patterns in species 495 richness. Ecography, 27, 268-272. 
Hawkins, B.A., Diniz-Filho, J.A.F, and Weis, A.E. (2005) The mid-domain effect and diversity gradients: is there anything to learn? American Naturalist. 166, E140-143

Hurlbert, A.H. \& White, E.P. (2005) Disparity between range map- and survey-based analyses of species richness: patterns, processes and implications. Ecology Letters, 8, 319-327.

500 Jetz, W. \& Rahbek, C. (2001) Geometric constraints explain much of the species richness pattern in African birds. Proceedings of the National Academy of Sciences of the United States of America, 98, 5661-5666.

Jetz, W. \& Rahbek, C. (2002) Geographic range size and determinants of avian species richness. Science, 297, 1548-1551.

505 Kelt, D.A., Taper, M.L. \& Meserve, P.L. (1995) Assessing the impact of competition on community assembly - a case-study using small mammals. Ecology, 76, 1283-1296.

Kerr, J.T., Perring, M. \& Currie, D.J. (2006) The missing Madagascan mid-domain effect. Ecology Letters, 9, 149-159.

Koleff, P. \& Gaston, K.J. (2001) Latitudinal gradients in diversity: real patterns and random models. Ecography, 24, 341-351.

Laurie, H. \& Silander, J.A. (2002) Geometric constraints and spatial pattern of species richness: critique of range-based null models. Diversity and Distributions, 8, 351-364.

Losos, J.B., Naeem, S. \& Colwell, R.K. (1989) Hutchinsonian ratios and statistical power. Evolution, 43, 1820-1826.

515 McCain, C.M. (2003) North American desert rodents: A test of the mid-domain effect in species richness. Journal of Mammalogy, 84, 967-980.

McClain, C.R. \& Etter, R.J. (2005) Mid-domain models as predictors of species diversity patterns: bathymetric diversity gradients in the deep sea. Oikos, 109, 555-566. 
Mora, C. \& Robertson, D.R. (2005) Causes of latitudinal gradients in species richness: A test with fishes of the Tropical Eastern Pacific. Ecology, 86, 1771-1782.

Pimm, S.L. \& Brown, J.H. (2004) Domains of diversity. Science, 304, 831-833.

Pineda, J. \& Caswell, H. (1998) Bathymetric species-diversity patterns and boundary constraints on vertical range distributions. Deep-Sea Research Part II-Topical Studies in Oceanography, 45, 83-101.

525 Rapoport, E.H. (1982) Areography: Geographical Strategies of Species. Pergamon Press, Oxford.

Rahbek, C.\& Graves, G.R. (2000) Detection of macro-ecological pattners in South American hummingbirds is affected by spatial scale. Proceedings of The Royal Society of London Series B, Biological Sciences, 267, 2259-2265.

530 Romdal, T.S., Colwell, R.K., \& Rahbek, C. (2005) The influence of band sum area, domain extent, and range sizes on the latitudinal mid-domain effect. Ecology, 86, 235-244.

Roughgarden, J. (1983) Competition and theory in community ecology. American Naturalist, 122, 583-601.

Sandel, B.S., \& McKone, M.J. (2006) Reconsidering null models of diversity: Do geometric constraints on species ranges necessarily cause a mid-domain effect? Diversity and Distributions, 12, 467-474.

Stevens, G.C. (1989) The latitudinal gradient in geographical range - how so many species coexist in the tropics. American Naturalist, 133, 240-256.

Toft, C.A. \& Shea ,P.J. (1983) Detecting community-wide patterns - estimating power strengthens statistical inference. American Naturalist, 122, 618-625. 
Vetaas, O.R. \& Grytnes, J.A. (2002) Distribution of vascular plant species richness and endemic richness along the Himalayan elevation gradient in Nepal. Global Ecology and Biogeography, 11, 291-301.

Watkins, Jr. J.E., Cardelus, C., Colwell R.K., \& Moran R.C. (2006) Species richness and distribution of ferns along an elevational gradient in Costa Rica. Journal of Botany, 93, 73-83.

Whittaker, R.J., Willis, K.J. \& Field, R. (2001) Scale and species richness: towards a general, hierarchical theory of species diversity. Journal of Biogeography, 28, 453-370.

Willig, M.R. \& Lyons, S.K. (1998) An analytical model of latitudinal gradients of species richness with an empirical test for marsupials and bats in the New World. Oikos, 81, 9398.

Zapata, F.A., Gaston, K.J. \& Chown, S.L. (2003) Mid-domain models of species richness gradients: assumptions, methods and evidence. Journal of Animal Ecology, 72, 677-690.

Zapata, F.A., Gaston, K.J. \& Chown, S.L. (2005) The mid-domain effect revisited. American Naturalist. 166, E144-148 

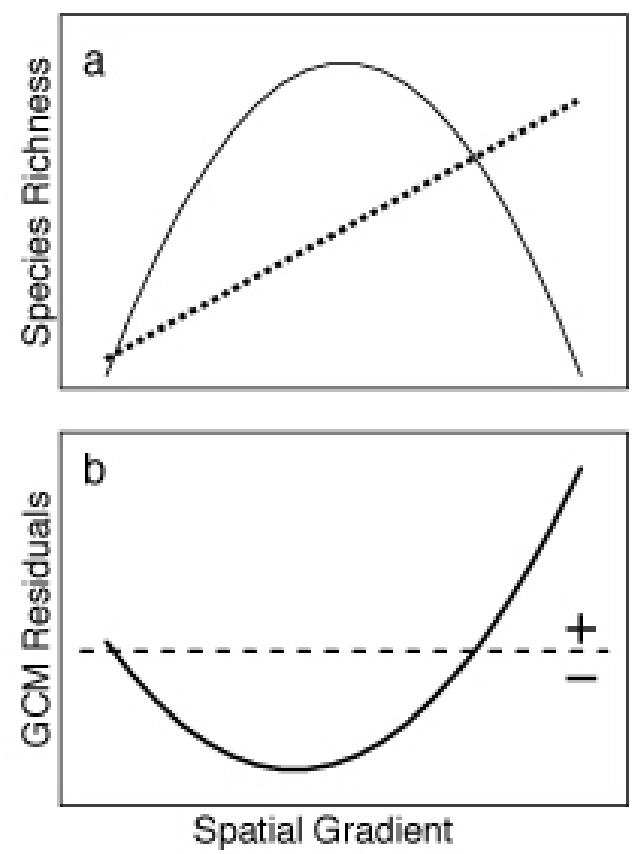

Figure 1: An example where species richness increases linearly across the domain (solid line, 1a). GCM predictions are hump-shaped pattern of richness (dotted line, 1a). Residuals from GCM expectations are J-shaped (1b). 


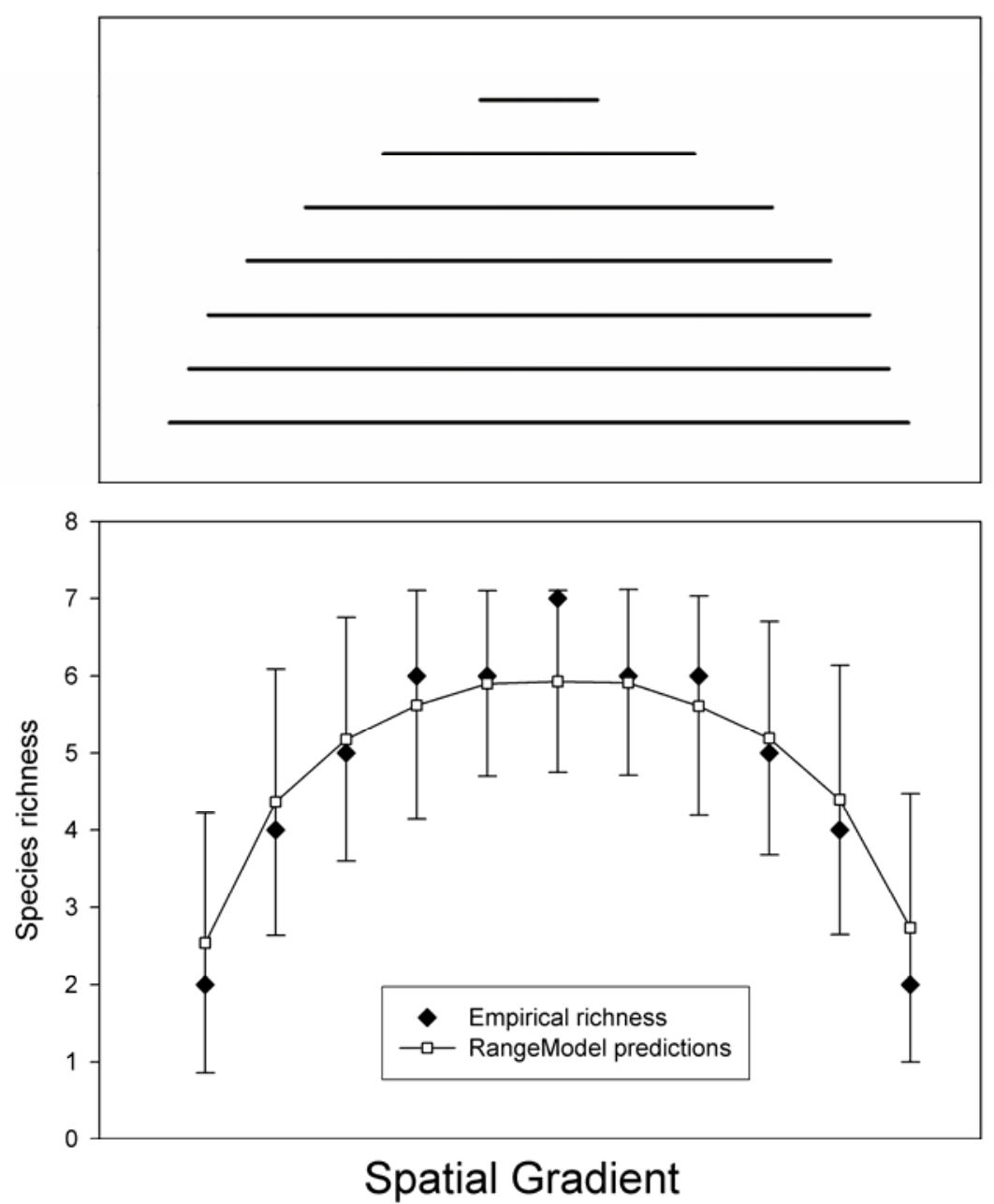

565 Figure 2: Example of richness varying across a one-dimensional domain in a manner consistent with the predictions of a geometric constraint model, but with ranges distributed in a distinctly non-random, perfectly nested manner. (A) Each line segment represents a species' range along a hypothetical spatial gradient. Ranges are spaced along the y-axis purely for illustrative purposes. (B) Empirical pattern of species richness and the pattern predicted by the random shuffling of ranges in (A) using Colwell's (2006) RangeModel software. Error bars represent 95\% confidence intervals based on 1000 simulations. 